OPEN ACCESS

Edited by:

Xiaoguang Zhou,

Beijing University of Posts and Telecommunications (BUPT), China

Reviewed by:

Miao Zhang

Monash University, Australia Jun Zhang,

Shanghai Normal University, China

${ }^{*}$ Correspondence:

Wanjun Zhang

wiz@henu.edu.cn

Specialty section:

This article was submitted to Bionics and Biomimetics,

a section of the journal

Frontiers in Bioengineering and

Biotechnology

Received: 16 April 2021

Accepted: 21 June 2021

Published: 06 August 2021

Citation:

Wang Y and Zhang W (2021) A Dense RNN for Sequential Four-Chamber

View Left Ventricle Wall Segmentation and Cardiac State Estimation.

Front. Bioeng. Biotechnol. 9:696227. doi: 10.3389/fbioe.2021.696227

\section{A Dense RNN for Sequential Four-Chamber View Left Ventricle Wall Segmentation and Cardiac State Estimation}

\author{
Yu Wang ${ }^{1}$ and Wanjun Zhang ${ }^{2 *}$ \\ ${ }^{1}$ Research Center for Physical Education Reform and Development, School of Physical Education, Henan University, Kaifeng, \\ China, ${ }^{2}$ Henan Key Laboratory of Big Data Analysis and Processing, School of Computer and Information Engineering, \\ Henan University, Kaifeng, China
}

The segmentation of the left ventricle (LV) wall in four-chamber view cardiac sequential image is significant for cardiac disease diagnosis and cardiac mechanisms study; however, there is no successful reported work on sequential four-chambered view LV wall segmentation due to the complex four-chamber structure and diversity of wall motion. In this article, we propose a dense recurrent neural network (RNN) algorithm to achieve accurately LV wall segmentation in a four-chamber view MRI time sequence. In the cardiac sequential LV wall process, not only the sequential accuracy but also the accuracy of each image matters. Thus, we propose a dense RNN to provide compensation for the first long short-term memory (LSTM) cells. Two RNNs are combined in this work, the first one aims at providing information for the first image, and the second RNN generates segmentation result. In this way, the proposed dense RNN improves the accuracy of the first frame image. What is more is that, it improves the effectiveness of information flow between LSTM cells. Obtaining more competent information from the former cell, frame-wise segmentation accuracy is greatly improved. Based on the segmentation result, an algorithm is proposed to estimate cardiac state. This is the first time that deals with both cardiac time-sequential LV segmentation problems and, robustly, estimates cardiac state. Rather than segmenting each frame separately, utilizing cardiac sequence information is more stable. The proposed method ensures an Intersection over Union (IoU) of $92.13 \%$, which outperforms other classical deep learning algorithms.

Keywords: four-chamber view cardiac, recurrent neural network, image segmentation, left ventricle wall, cardiac state estimation

\section{INTRODUCTION}

The sequential segmentation of the left ventricle (LV) wall in a four-chamber view MRI image plays an important role in clinical disease diagnosis and physiological mechanism research works. Compared with a two-chamber view cardiac image, a four-chamber view image has distinctive advantages in cardiac assessment. For example, it is needed to compare the four chambers of cardiac in size and contractility to diagnose congenital heart disease (Copel et al., 1987); an LV sequence image can be used to evaluate the LV wall motion, which has been used to evaluate the risk of heart 
failure (Konstam et al., 2011); Many studies have focused on the mechanisms of excitable tissue in cardiac based on the morphological feature of an LV wall (Constantino et al., 2010). Furthermore, a four-chamber view sequence is used to measure the relationship between left ventricular diastolic dysfunction and exercise intolerance in obese heart failure with preserving (Samuel et al., 2021), the cardiac state estimate is critical for the assessment of cardiac function and morphology. Thus, it is desired to propose a method to solve the sequential LV wall segmentation problem in a four-chamber view MRI image.

\subsection{Challenges of LV Segmentation}

However, the automatic segmentation of LV wall in four-chamber view MRI images is still a challenge, as shown in Figure 1, (1) the complex structure of four-chamber view MRI image makes it hard to separate LV wall with other tissues. Figure 1A also shows that, compared with the two-chamber view cardiac image, the influence of the right ventricle (RV) and right atrium makes it harder to segment the LV wall. The similarity in intensity and structure feature with RV wall also increased the difficulty of LV wall segmentation. (2) High segmentation accuracy is desired in clinical for wall motion evaluation. Figure $\mathbf{1 B}$ shows LV wall changes at contraction state and diastole state. The difference between neighbor frames is small, which needs high accuracy on distinguishing contraction state and diastole state. (3) Segmentation error evaluation problem. Figure 1C shows that, the error area and IoU of the two error detection illustration examples are the same; however, in sequential image processing progress, their effect on clinical evaluation is different and may lead to misdiagnosis. Thus, a method is needed to be proposed to deal with time-sequential cardiac images and to achieve both frame-wise and sequence-wise accuracy.

\subsection{Related Works}

\subsubsection{Automated Cardiac Segmentation Methods}

Unfortunately, there is no successful reported research work focusing on automatic sequential LV wall segmentation due to the challenges mentioned above. Most research studies focus on the assessment of LV sequence parameters (such as LV wall thickness, area, and so on) and the LV wall segmentation of a single frame. Recently, the deep learning method is widely used in medical image processing. An anatomically constrained neural network (ACNN) based cardiac image enhancement and segmentation method is proposed in Oktay et al. (2018). The method works well on MRI images. In most previous segmentation works (Tran, 2016; Oktay et al., 2018; Khened et al., 2019), each frame of the MRI sequence is processed independently. To achieve continuity of segmentation or quantification and take advantage of the sequence information, a correlation between different frame must be established. In the language generative deep learning method, sequence generate adversarial nets (SGAN) proposed in Yu et al. (2017) performs well in sequence processing and achieves a good result; however, this model can-not get a satisfactory result when it comes to sequence image segmentation.

\subsubsection{Deep Learning Architectures for Medical Image Segmentation}

In the context of medical image segmentation method, convolutional neural networks (CNN)-based works have shown great potential in recent studies (Renard et al., 2020). In methods (Avendi et al., 2016; Ngo et al., 2017; Kasinathan et al., 2019; Ma and Yang, 2020), CNN and traditional methods (such as level set method and deformable models) are combined to achieve good segmentation accuracy. Fully convolutional

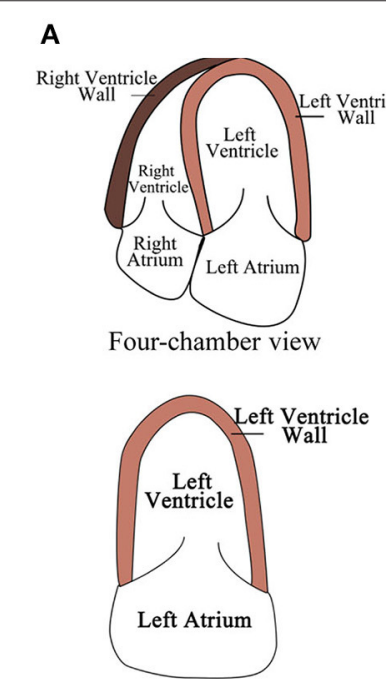

Two-chamber view
B
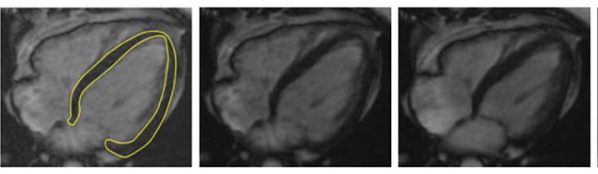

Contraction
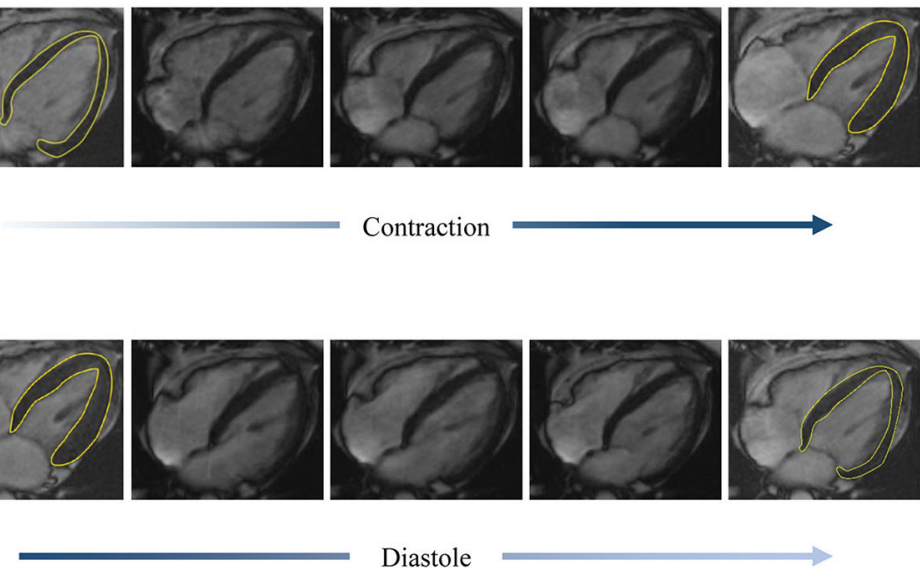

C
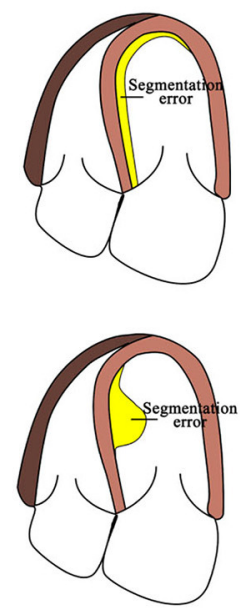

FIGURE 1 | Challenges for four-chamber view MRI image segmentation. (A) The structure of the four-chamber view cardiac image is more complex than that of the two-chamber view. (B) The LV wall difference between frames caused by cardiac motion is small, thus high accuracy is needed. (C) Detection error with the same area but the different effect to its subsequent frames. 
networks (FCN) (Long et al., 2015) and U-net (Ronneberger et al., 2015) have achieved remarkable success in the image segmentation problem. Compared with original U-net, attention modules combined U-net (Dong et al., 2018; Schlemper et al., 2019), self-guided attention U-net (Ashish and Jose, 2021) has resulted in enhanced models for pixel-wise segmentation tasks. Based on U-net, a recurrent convolutional neural network (RCNN) is proposed in Alom et al. (2018). The feature accumulation in RCNN ensures better feature representation for segmentation tasks. In Peng et al. (2020), a deep snake method is proposed to segment image by controlling the movement of object boundaries. In Xie et al. (2020), a PolarMask method is proposed and contour of instance in a polar coordinate is predicted. These two methods are good at segment instance with less concave property.

\section{Recurrent Neural Network}

Recurrent Neural Network (RNN) (Zaremba et al., 2014) is specialized in processing sequential data. Long short term memory (LSTM) cell (Greff et al., 2015) or gated recurrent unit (GRU) Cho et al. (2014) are combined in RNN to transfer information. Promising results have been achieved by RNN or RNN variants in speech processing (Karita et al., 2019; Li et al., 2019), text generation (Pawade et al., 2018; Yang et al., 2019), classification (Premkumar et al., 2020), and image processing (Yao et al., 2019; Zhou et al., 2020). RNN has been used in Xue et al. (2017) regional wall thicknesses of LV.

Convolutional networks, such as FCN and U-net, focus on the problem of single-frame image processing. Compared with other neural networks, RNN is good at dealing with sequential image processing problems. The cardiac in MRI sequence contracts and diastole continuously. To make the best of information between frames, we choose RNN to achieve the LV wall segmentation goal.

\subsection{Contributions}

We proposed a dense RNN method to deal with the challenges and overcome the shortage of existing methods mentioned above. The details of highlights are as follows:

(1) It is the first time that an RNN successfully deals with a timesequential cardiac segmentation problem. Existing methods focus on single frame segmentation or difference slice sequential segmentation. In this article, the time-sequential frames belong to one slice of cardiac, but with different cardiac state, which is able to observe cardiac structure change. Rather than segment a single image, segment sequence takes advantage of the relationship between frames, which makes it better to solve the complex structures and wall motion problems.

(2) A dense RNN is proposed to improve the effectiveness of the information transmitted between LSTM cells and achieve frame-wise accuracy. In RNN, the first frame acquires no hidden information, but its LSTM output is transmitted to the rest of the frames and plays a most important role. The proposed dense RNN contains two RNNs. The first RNN aims at providing dense hidden information for the first LSTM cell in the second RNN. The second RNN receives the

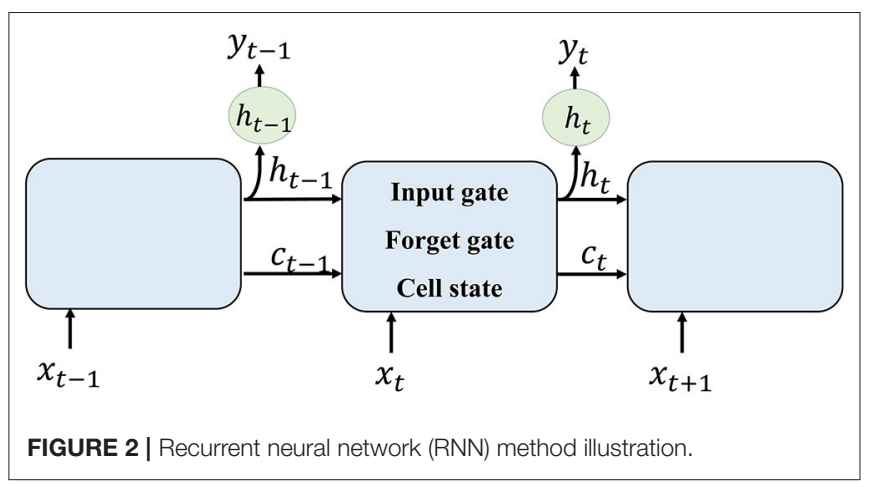

hidden information and generates the segmentation result. This not only contributes to improving the accuracy of the first image but also makes the output of the first LSTM cell contain more useful information. It is the same with the subsequent cells. Thus, frame-wise accuracy is improved.

(3) Based on the LV wall segmentation result, an algorithm is proposed to determine the cardiac state. The algorithm uses mean cardiac change difference instead of the difference between frames, which is more robust. As the change of neighbor frames is relatively small, we first calculate the difference between two neighbor frames and then use the mean difference to determine the cardiac state.

This article is organized as follows: first, the background of cardiac LV segmentation and the existing method are introduced; second, the proposed method will be illustrated; third, the experimental result will be analyzed.

\section{METHOD}

The proposed network mainly contains two RNNs. In this section, RNN is first introduced. And then, the proposed dense $\mathrm{RNN}$ is described.

\subsection{Recurrent Neural Network}

Recurrent neural network is good at dealing with sequential information. It consists of several LSTM cells. Each unit receives a hidden layer from the former cell, together with the current input, the output layer is generated.

The method of RNN is shown in Figure 2. Each LSTM cell receives information from the previous cell and transmits information to the next one. For an input image sequence $x_{1}, x_{2}, \ldots, x_{n}$, each $x_{t}$ input into an LSTM cell. In general, an LSTM cell contains an input gate that generates $i_{t}$, a forget gate that generates $f_{t}$, and a memory vector $c_{t}$. The relationship between these gates and the vector of LSTM can be denoted as follows:

$$
\begin{aligned}
f_{t} & =\operatorname{sigm}\left(W_{f} \cdot\left[h_{t-1}, x_{t}\right]+b_{f}\right) \\
i_{t} & =\operatorname{sigm}\left(W_{i} \cdot\left[h_{t-1}, x_{t}\right]+b_{i}\right) \\
\widetilde{c_{t}} & =\tanh \left(W_{o} \cdot\left[h_{t-1}, x_{t}\right]+b_{o}\right)
\end{aligned}
$$




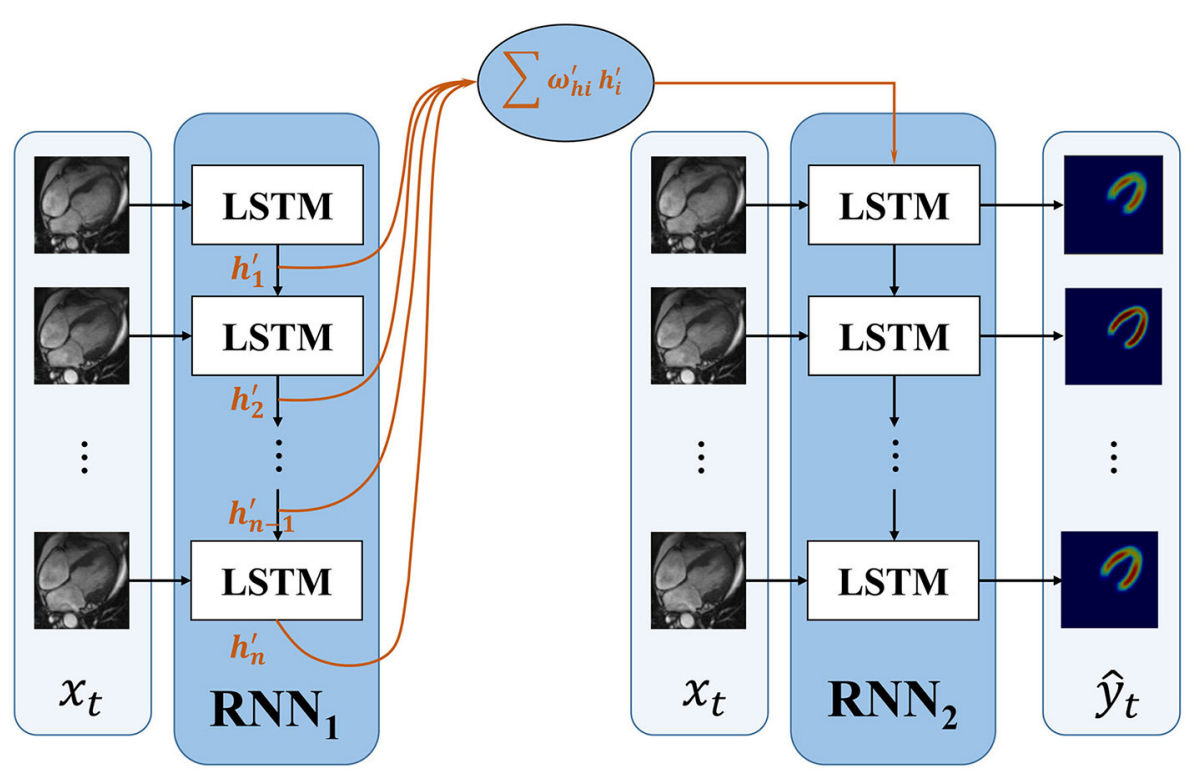

FIGURE 3 | The framework of the proposed dense RNN

where $h_{t-1}$ is the hidden layer of the former cell and $b$ is the bias factor. Then, the memory cell of LSTM can be calculated as follows:

$$
c_{t}=f_{t} * c_{t-1}+i_{t} * \tilde{c_{t}}
$$

where $*$ is convolution transform. The output $o_{t}$ and the hidden layer to the next LSTM are given by the following :

$$
\begin{aligned}
& o_{t}=\operatorname{sigm}\left(W_{o} \cdot\left[h_{t-1}, x_{t}\right]+b_{o}\right) \\
& h_{t}=o_{t} \odot \tanh \left(c_{t}\right)
\end{aligned}
$$

To formulate the segmentation result of one input $x_{t}$, the current cell receives hidden layer $h_{t-1}$ and memory vector $c_{t-1}$ from former cell. The forget gate selects information that should be abandoned. Combining $h_{t-1}$ and current input $x_{t}$, the input gate selects new information that should be kept and generates a new cell state. The output of one cell is generated by the current input and formerly hidden layer.

\subsection{Dense RNN}

As illustrated in section RNN and shown in Figure 2, an LSTM cell $L_{i}$ receives a hidden layer and memory cell from LSTM cell $L_{i-1}$, together with input image $x_{i}$, its segmentation image is generated. The first image in the sequence receives no information from the former cell. Thus, the accuracy of the first image is lower than the others. The hidden layer information and memory cell it flows to its following frames contain lower effective information.

To improve the accuracy of the first frame and make the information transmitted between frames more efficient, we propose an improved RNN. The proposed dense RNN is constituted by two RNNs. As shown in Figure 3, dense generator, main $\mathrm{RNN}\left(R N N_{2}\right.$ in Figure 3$)$ is used to generate segmentation results, while compensation $\operatorname{RNN}\left(R N N_{1}\right.$ in Figure 3) provides compensation for the first LSTM cell in main RNN. With this compensation, the first frame acquires more information and makes the information flow in the main RNN more effective.

The first LSTM cell plays an important role in RNN. Its output information transmits to the subsequent LSTM cells; however, the first LSTM receives no hidden information, which means $h_{0}=0$. The only input information it deals is $x_{1}$. In this proposed dense RNN, the input hidden layer of the first LSTM cell in $R N_{2}$ can be denoted as follows:

$$
h_{0}=\sum_{i=1}^{n} \omega_{h i}^{\prime} h_{n}^{\prime}
$$

where $h_{1}^{\prime}, h_{2}^{\prime}, \ldots, h_{n}^{\prime}$ denote the output hidden layer of each LSTM in $R N N_{1}$. The proposed dense RNN allows the network itself to choose the proper input hidden layer for the main RNN. The weight $\omega_{h i}^{\prime}$ in Equation 4 is trained by the network. With the weighted input hidden layer, the accuracy of the first frame is improved and the output of the first LSTM in the main RNN contains more useful information. In this way, the segmented accuracy of the subsequent frames is also improved.

The loss function is calculated by measuring the difference of ground truth and the output of the second RNN:

$$
L=\sum_{t=1}^{T}\left(-y_{t} \log \hat{y}_{t}-\left(1-y_{t}\right) \log \left(1-\hat{y}_{t}\right)\right)
$$

where $y_{t}$ is the ground truth of frame $t$ and $\hat{y}_{t}$ is the output of $\mathrm{RNN}_{2}$. 


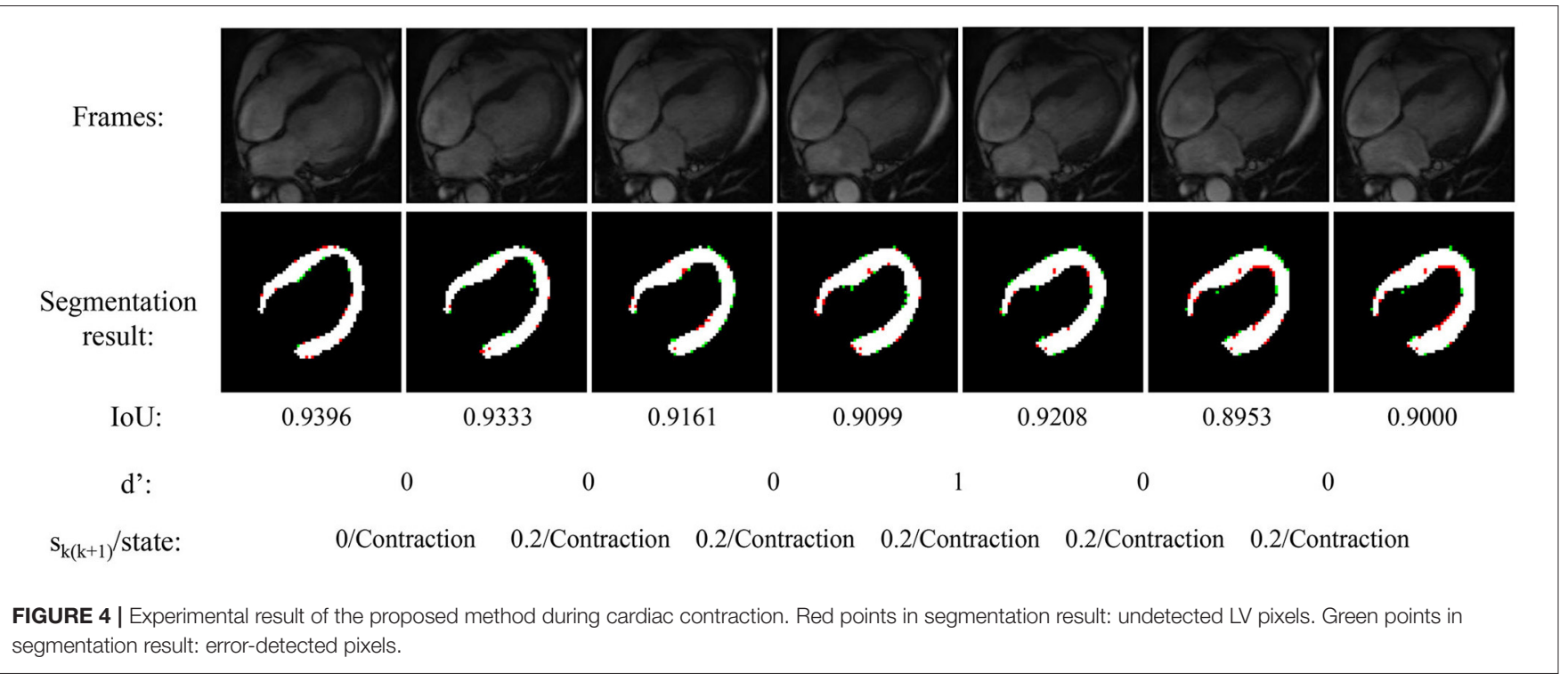

\subsection{Cardiac State Estimation}

The LV wall becomes thick during the contraction state and becomes thin during the diastole state. However, the change is not obvious for adjacent frames. In this article, we first calculate the difference between two adjacent frames as a reference. The difference of frame $k$ and frame $k+1$ is defined by comparing the area of LV wall using Equation 6.

$$
d_{k(k+1)}^{\prime}= \begin{cases}1, & \text { if } \operatorname{Area}\left(f_{k}\right)>\operatorname{Area}\left(s_{k+1}\right) \\ 0, & \text { else }\end{cases}
$$

where $\operatorname{Area}_{\left(f_{k}\right)}$ is the $\mathrm{LV}$ wall segmentation result of the area of the kth frame. Then, the cardiac state is estimated by the following equation:

$$
s_{k(k+1)}=\frac{1}{N} \sum_{i=k-N / 2}^{k+N / 2-1} d_{i(i+1)}^{\prime}
$$

With the result of equation $7, s_{k(k+1)}<0.5$ means that the cardiac frame is in a state of diastole, or else it is in a state contraction. By using Equation 7, the incorrect estimation by only two adjacent frames was reduced.

\section{EXPERIMENTAL RESULTS AND DISCUSSION}

\subsection{Dataset and Setting}

The proposed method is tested using 137 groups of four-chamber view MRI cardiac images from 137 patients. Each group contains 18 continuous frames of the image, and every group contains frames of contraction and diastole cardiac states. The images are resized to $64 \times 64$. The ground truth of the LV wall is manually marked by doctors. We adopt 130 groups of images for training and the other 7 groups for testing. During training process, we use different frames as start frames to improve the robustness of the networks. The proposed network is implemented based on PyCharm and performed on NVIDIA Tesla P100.

\subsection{Evaluation Metrics}

The proposed method performs better than SeqGAN (Yu et al., 2017) and CNN method. The segmentation result is evaluated using the IoU factor. The IoU factor is obtained by $I o U=\frac{S \cap G}{S \cup G}$, where $S$ is the segmentation result and $G$ is the ground truth. $\cap$ and $U$ is the action of intersection and union.

\subsection{Generation Performance}

Figure 4 shows the experimental result of the proposed method. The frames in this sequence are during a contraction state, and the LV wall changes from thin to thick. The mean IoU of these frames is $91.64 \%$. Though the changes between frames are little, the segmentation result reflex the change of LV wall thickness. Figure 5 shows the segmentation result during cardiac diastole. It can be seen from Figures $\mathbf{4}$ to $\mathbf{5}$ that the proposed method can obtain accurate diastole and contraction cardiac LV wall.

Figure 4 also illustrated the cardiac state method. All the frames are in a period of contraction. The difference of adjacent frames $d^{\prime}$ incorrect estimate two frames as diastole with value 1. After the mean value calculated by equation 7 , the incorrect estimate is corrected.

Figure 6 shows the IoU of the proposed method and other methods at different training interactions. The proposed method reaches an IoU of $92.13 \%$, while the RNN method without dense net gets a result of $38.92 \%$. We also use Unet (Ronneberger et al., 2015), a classic CNN deep learning method in image segmentation, to segment the LV wall. After 2000 times of interactions, the IoU reaches $61.75 \%$. Compared with the other 


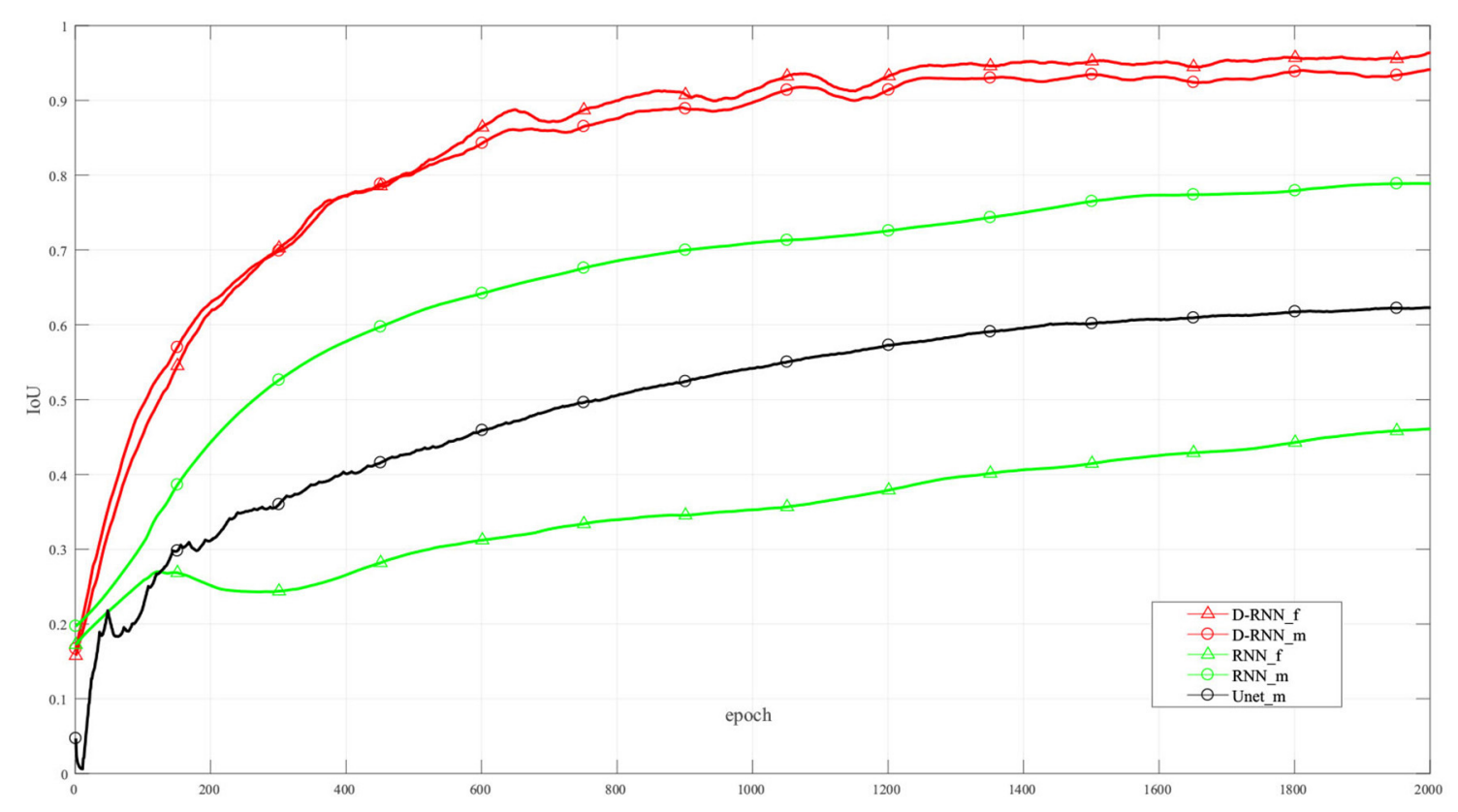

FIGURE 5 | Segmentation result of the proposed method during cardiac diastole.

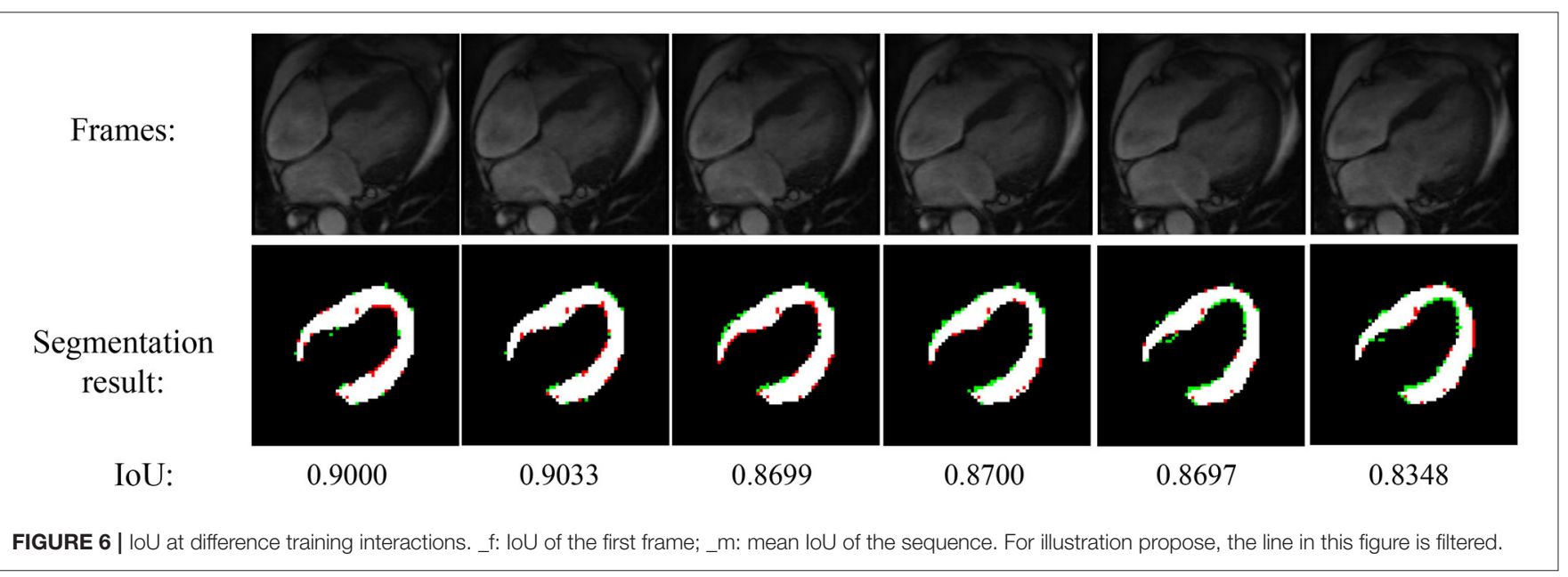

method, the proposed method highly improved the mean sequential segmentation accuracy.

The dense compensation for an RNN proposed in this method greatly improved the accuracy of the first frame in the sequence. Thus, the proposed method makes the hidden information flow in different LSTM cells more effectively, and the mean IoU is improved. It can be seen from Figure 6, the NoDense curve, that although the mean IoU by NoDense network is increasing with the increasing interaction, the IoU of the first frame remains unsatisfied. Each frame in the image sequence needed to be segmented with a promising result. The proposed dense RNN in DL-GAN works improves the mean accuracy and also ensures the accuracy of each frame. It can be seen from the DL-GAN curve in Figure 6 that the IoU factor of the first frame increases along with the mean IoU of the sequence. The mean accuracy is increased by $55.21 \%$ than the NoDense network.

\section{CONCLUSION}

In this article, a dense RNN method is proposed to segment the $\mathrm{LV}$ wall in a sequential four-chamber view MRI image. With the dense RNN, the segmentation accuracy of each frame in the sequence is guaranteed, and the accuracy of the first frame is greatly improved. The network reaches an IoU of $92.13 \%$, which indicates the proposed method has prospects in cardiac disease diagnosis and cardiac mechanism analysis. 


\section{DATA AVAILABILITY STATEMENT}

The original contributions presented in the study are included in the article/supplementary material, further inquiries can be directed to the corresponding author/s.

\section{AUTHOR CONTRIBUTIONS}

YW contributed to the conception of the study and data analysis. WZ performed the experiment and wrote the manuscript.

\section{REFERENCES}

Alom, M. Z., Hasan, M., Yakopcic, C., Taha, T. M., and Asari, V. K. (2018). Recurrent residual convolutional neural network based on u-net (r2u-net) for medical image segmentation. arXiv preprint arXiv:1802.06955. doi: 10.1109/NAECON.2018.8556686

Ashish, S., and Jose, D. (2021). Multi-scale self-guided attention for medical image segmentation. IEEE J. Biomed. Health Inform. 25, 121-130. doi: 10.1109/JBHI.2020.2986926

Avendi, M., Kheradvar, A., and Jafarkhani, H. (2016). A combined deeplearning and deformable-model approach to fully automatic segmentation of the left ventricle in cardiac mri. Med. Image Anal. 30,108-119. doi: 10.1016/j.media.2016.01.005

Cho, K., van Merriënboer, B., Gulcehre, C., Bougares, F., Schwenk, H., and Bengio, Y. (2014). Learning phrase representations using rnn encoder-decoder for statistical machine translation. arxiv. Available online at: https://arxiv.org/abs/ 1406.1078v3. doi: 10.3115/v1/D14-1179

Constantino, J., Long, Y., Ashihara, T., and Trayanova, N. A. (2010). Tunnel propagation following defibrillation with icd shocks: hidden postshock activations in the left ventricular wall underlie isoelectric window. Heart Rhythm 7, 953-961. doi: 10.1016/j.hrthm.2010.03.026

Copel, J. A., Pilu, G., Green, J., Hobbins, J. C., and Kleinman, C. S. (1987). Fetal echocardiographic screening for congenital heart disease: the importance of the four-chamber view. Am. J. Obstet. Gynecol. 157, 648-655. doi: 10.1016/S0002-9378(87)80022-4

Dong, N., Yaozong, G., Li, W., and Dinggang, S. (2018). "Asdnet: attention based semi-supervised deep networks for medical image segmentation," in International Conference on Medical Image Computing and Computer-Assisted Intervention (Granada: Springer), 370-378.

Greff, K., Srivastava, R., Koutník, J., Steunebrink, B., and Schmidhuber, J. (2015). Lstm: a search space odyssey. IEEE Trans. Neural Netw. Learn. Syst. 28, 2222-2232. doi: 10.1109/TNNLS.2016.2582924

Karita, S., Chen, N., Hayashi, T., Hori, T., Inaguma, H., Jiang, Z., et al. (2019). “A comparative study on transformer vs rnn in speech applications," in 2019 IEEE Automatic Speech Recognition and Understanding Workshop (ASRU) (Sentosa), 449-456.

Kasinathan, G., Jayakumar, S., Gandomi, A. H., Ramachandran, M., Fong, S. J., and Patan, R. (2019). Automated 3-d lung tumor detection and classification by an active contour model and cnn classifier. Expert. Syst. Appl. 134, 112-119. doi: 10.1016/j.eswa.2019.05.041

Khened, M., Kollerathu, V. A., and Krishnamurthi, G. (2019). Fully convolutional multi-scale residual densenets for cardiac segmentation and automated cardiac diagnosis using ensemble of classifiers. Med. Image Anal. 51, 21-45. doi: 10.1016/j.media.2018.10.004

Konstam, M. A., Kramer, D. G., Patel, A. R., Maron, M. S., and Udelson, J. E. (2011). Left ventricular remodeling in heart failure: current concepts in clinical significance and assessment. JACC Cardiovasc. Imaging 4, 98-108. doi: 10.1016/j.jcmg.2010.10.008

Li, J., Zhao, R., Hu, H., and Gong, Y. (2019). "Improving rnn transducer modeling for end-to-end speech recognition," in 2019 IEEE Automatic Speech Recognition and Understanding Workshop (ASRU) (Singapore: IEEE), 114-121.
Both authors contributed to the article and approved the submitted version.

\section{FUNDING}

This work was funded by Key R\&D and Promotion Projects of Henan Province (No. 212102311014), National Natural Science Foundation of China (No. 62002100), Henan Province Philosophical Social Science Planned Item (No. 2019CTY016), and the Education Department of Henan province of Humanities and Social Science Project (No. 2020-ZDJH-039).

Long, J., Shelhamer, E., and Darrell, T. (2015). Fully convolutional networks for semantic segmentation. IEEE Trans. Pattern Anal. Mach. Intell. 39, 640-651. doi: 10.1109/CVPR.2015.7298965

Ma, J., and Yang, X. (2020). "Combining cnn and hybrid active contours for head and neck tumor segmentation in ct and pet images," in 3D Head and Neck Tumor Segmentation in PET/CT Challenge (Springer), 59-64.

Ngo, T. A., Lu, Z., and Carneiro, G. (2017). Combining deep learning and level set for the automated segmentation of the left ventricle of the heart from cardiac cine magnetic resonance. Med. Image Anal. 35, 159-171. doi: 10.1016/j.media.2016.05.009

Oktay, O., Ferrante, E., Kamnitsas, K., Heinrich, M., Bai, W., Caballero, J., et al. (2018). Anatomically constrained neural networks (acnns): application to cardiac image enhancement and segmentation. IEEE Trans. Med. Imaging 37, 384-395. doi: 10.1109/TMI.2017.2743464

Pawade, D., Sakhapara, A. M., Jain, M., Jain, N., and Gada, K. (2018). Story scrambler-automatic text generation using word level rnn-lstm. Int. J. Inform. Technol. Comput. Sci. 10, 44-53. doi: 10.5815/ijitcs.2018. 06.05

Peng, S., Jiang, W., Pi, H., Li, X., Bao, H., and Zhou, X. (2020). “Deep snake for real-time instance segmentation," in Proceedings of the IEEE/CVF Conference on Computer Vision and Pattern Recognition (Seattle, WA), 8533-8542.

Premkumar, K. A. R., Bharanikumar, R., and Palaniappan, A. (2020). Riboflow: using deep learning to classify riboswitches with $\sim 99 \%$ accuracy. Front. Bioeng. Biotechnol. 8:808. doi: 10.3389/fbioe.2020.00808

Renard, F., Guedria, S., De Palma, N., and Vuillerme, N. (2020). Variability and reproducibility in deep learning for medical image segmentation. Sci. Rep. 10, 1-16. doi: 10.1038/s41598-020-69920-0

Ronneberger, O., Fischer, P., and Brox, T. (2015). "U-net: Convolutional networks for biomedical image segmentation," in International Conference on Medical Image Computing and Computer-Assisted Intervention (Cham: Springer), 234-241.

Samuel, T. J., Kitzman, D., Haykowsky, M., Upadhya, B., Brubaker, P., Nelson, M., et al. (2021). Left ventricular diastolic dysfunction and exercise intolerance in obese heart failure with preserved ejection fraction. Am. J. Physiol. Heart Circ. Physiol. 320, H1535- 1542. doi: 10.1152/ajpheart.00610.2020

Schlemper, J., Oktay, O., Schaap, M., Heinrich, M., Kainz, B., Glocker, B., et al. (2019). Attention gated networks: learning to leverage salient regions in medical images. Med. Image Anal. 53, 197-207. doi: 10.1016/j.media.2019.01.012

Tran, P. V. (2016). A fully convolutional neural network for cardiac segmentation in short-axis mri. arXiv preprint arXiv:1604.00494.

Xie, E., Sun, P., Song, X., Wang, W., Liu, X., Liang, D., et al. (2020). "Polarmask: Single shot instance segmentation with polar representation," in Proceedings of the IEEE/CVF Conference on Computer Vision and Pattern Recognition (Seattle, WA), 12193-12202.

Xue, W., Nachum, I. B., Pandey, S., Warrington, J., Leung, S., and Li, S. (2017). "Direct estimation of regional wall thicknesses via residual recurrent neural network," in Information Processing in Medical Imaging, eds M. Niethammer, M. Styner, S. Aylward, H. Zhu, I. Oguz, P. -T. Yap, and D. Shen (Boone, NC: Springer International Publishing), 505-516.

Yang, Z., Guo, X., Chen, Z., Huang, Y., and Zhang, Y. (2019). Rnnstega: Linguistic steganography based on recurrent neural networks. IEEE 
Trans. Inform. Forensics Secur. 14, 1280-1295. doi: 10.1109/TIFS.2018. 2871746

Yao, H., Zhang, X., Zhou, X., and Liu, S. (2019). Parallel structure deep neural network using cnn and rnn with an attention mechanism for breast cancer histology image classification. Cancers 11:1901. doi: 10.3390/cancers111 21901

Yu, L., Zhang, W., Wang, J., and Yu, Y. (2017). "Seqgan: sequence generative adversarial nets with policy gradient," in Proceedings of the Thirty-First AAAI Conference on Artificial Intelligence (San Francisco, CA: AAAI-2017), 2852-2858.

Zaremba, W., Sutskever, I., and Vinyals, O. (2014). Recurrent neural network regularization. arXiv preprint arXiv: 1409.2329

Zhou, X., Li, Y., and Liang, W. (2020). Cnn-rnn based intelligent recommendation for online medical pre-diagnosis support. IEEE/ACM Trans. Comput. Biol. Bioinform. 18, 912-921. doi: 10.1109/TCBB.2020. 2994780
Conflict of Interest: The authors declare that the research was conducted in the absence of any commercial or financial relationships that could be construed as a potential conflict of interest.

Publisher's Note: All claims expressed in this article are solely those of the authors and do not necessarily represent those of their affiliated organizations, or those of the publisher, the editors and the reviewers. Any product that may be evaluated in this article, or claim that may be made by its manufacturer, is not guaranteed or endorsed by the publisher.

Copyright $\odot 2021$ Wang and Zhang. This is an open-access article distributed under the terms of the Creative Commons Attribution License (CC BY). The use, distribution or reproduction in other forums is permitted, provided the original author(s) and the copyright owner(s) are credited and that the original publication in this journal is cited, in accordance with accepted academic practice. No use, distribution or reproduction is permitted which does not comply with these terms. 